\title{
Structure and strategy in encoding simplified graphs
}

\author{
DIANE J. SCHIANO and BARBARA TVERSKY \\ NRC/NASA Ames Research Center, Moffett Field, California \\ and Stanford University, Stanford, California
}

\begin{abstract}
Tversky and Schiano (1989) found a systematic bias toward the $45^{\circ}$ line in memory for the slopes of identical lines when embedded in graphs, but not in maps, suggesting the use of a cognitive reference frame specifically for encoding meaningful graphs. The present experiments explore this issue further using the linear configurations alone as stimuli. Experiments 1 and 2 demonstrate that perception and immediate memory for the slope of a test line within orthogonal "axes" are predictable from purely structural considerations. In Experiments 3 and 4, subjects were instructed to use a diagonal-reference strategy in viewing the stimuli, which were described as "graphs" only in Experiment 3. Results for both studies showed the diagonal bias previously found only for graphs. This pattern provides converging evidence for the diagonal as a cognitive reference frame in encoding linear graphs, and demonstrates that even in highly simplified displays, strategic factors can produce encoding biases not predictable solely from stimulus structure alone.
\end{abstract}

Graphs are symbolic as well as pictorial stimuli, requiring both perceptual and conceptual encoding for comprehension and memory. Initial perceptual encoding processes for any visual stimulus include separating figure from ground and perceptual grouping. In addition, there is evidence that reference points or frames can be used to anchor encoding of visual stimuli, resulting in systematic distortions (e.g., Nelson \& Chaiklin, 1980; Rosch, 1975; Taylor, 1961; Tversky, 1981; Tversky \& Schiano, 1989). Whether the reference frame is physically present or implied in the stimulus display, stimulus elements tend to become organized with respect to it. For example, in the perceptual literature, it is well known that the perceived orientation of an isolated test line tends to be drawn toward the nearest cardinal axis (Andrews, 1967; Bouma \& Andriessen, 1968; see Howard, 1982). Similarly, many visual illusions (including, e.g., the well-known MüllerLyer, Delbouef, and Poggendorf effects) appear to reflect distortions with respect to either reference elements in the display (see Coren \& Girgus, 1978) or the cardinal axes (e.g., Weintraub, Krantz, \& Olson, 1980).

For any given pictorial stimulus, a number of potential reference frames may be available. Perceptual references, specified by visual information, may include the relative locations of other figures in the display, the horizontal and vertical axes of the world, and the sides of the page by which the picture is framed. Cognitive reference frames have been amply demonstrated in the "cognitive

This research was supported in part by AFSOR Grant 89-0076 to Stanford University. We are grateful to James Chumbley, Pierre Jolicouer, Dana Kay Smith, and Peter Wenderoth for helpful comments on earlier versions of this paper. Correspondence should be addressed to Diane J. Schiano, MS 262-3, NASA Ames Research Center, Moffett Field, CA 94035. map" literature; assimilation to such map-specific references as landmarks (Hirtle \& Jonides, 1985; Sadalla, Burroughs, \& Staplin, 1980), boundary lines (McNamara, 1986; Stevens \& Coupe, 1978), and grid street patterns (Byme, 1979; Moar \& Bower, 1983) is commonly found. For maps, the canonical axes (i.e., north-south, eastwest) also constitute cognitive reference frames. Distortion toward the nearest canonical axis in remembered maps has been demonstrated by Tversky (1981).

In the case of $x-y$ graphs of linear functions, another potential cognitive reference frame suggests itself: the implicit $45^{\circ}$ diagonal. This identity line, where $x=y$, permits an assessment "at a glance" of the relative magnitude of a plotted effect or the degree of fit between predicted and obtained results. Tversky and Schiano (1989) reported research in which single lines or linear scatterplots of points were embedded in orthogonal axes and labeled as graphs. The functions depicted by the graphs were meaningful, and to induce a natural comprehension set, the subject's task involved either drawing the "function line"' in a response graph or verbally describing the depicted relationship. The slope of the function line was varied, and the data of interest were the slopes of the reproduced lines. The results of two experiments showed a systematic distortion of the slope of the response lines toward the implicit $45^{\circ}$ line. We argued that these results suggest that the $45^{\circ}$ line is a salient cognitive reference frame specifically used to encode and remember linear functions in graphs. Some evidence to support this view was given by a comparison condition in which stimulus displays identical to those presented as graphs to one group of subjects were presented as maps to another group. The "axes" were given street names, and the "function line" was portrayed as a bike path to a specified location. The results for the maps differed dramati- 
cally from those for the graphs; no evidence of a bias toward the diagonal was found (if anything, the bias was toward the axes). Taken together, this pattern of resuits suggests that the diagonal is strategically chosen as a reference line for encoding graphs but not other kinds of visual displays, even those highly similar in structure to graphs.

While the study of cognitive maps has become a large field of research in its own right, surprisingly little psychological work has been performed on systematic errors in understanding and remembering graphs (Poulton, 1985, and Bryant \& Somerville, 1986, are notable exceptions). Indeed, though discussions of potential sources of error in reading graphs have become increasingly popular (e.g., Cleveland, 1985; Kolata, 1984; Tufte, 1983), systematic work on how graphs are perceived and remembered has only just begun. Much of this research has been performed by statisticians, such as Cleveland and colleagues (e.g., Cleveland \& McGill, 1985, 1986), who have attempted to explain relative accuracy of various judgments thought to be involved in graph perception (e.g., line length and height, angle estimations) in terms of psychophysical principles (i.e., the size of the exponent of the power law). Cognitive factors involved in graph comprehension and memory clearly require further investigation.

This paper describes a series of experiments designed to confirm and extend Tversky and Schiano's initial findings on the use of the $45^{\circ}$ line as a cognitive reference frame for encoding and remembering linear functions in graphs. In that earlier work, the linear configuration was embedded in a complex symbolic display, and additional tasks were imposed to ensure that the stimuli were convincing as graphs or maps, respectively. However, no attempt was made to examine how the linear configuration per se (i.e., the three converging line segments without any additional information or symbolic significance) was perceived and remembered. Nor was the issue of strategic control of cognitive reference frames directly addressed. In the present research, four experiments were performed using the same linear configurations as stimuli. In the first two experiments, perception and memory for these "meaningless" displays were examined. If the diagonal bias we observed for meaningful graphs reflects assimilation toward a cognitive reference frame used specifically for encoding the slopes of linear functions in graphs (and not for encoding meaningless linear displays), then a very different pattern of results might be expected for these stimuli: the systematic bias toward the diagonal either should not be found or should be considerably reduced. In the next two experiments, the strategic nature of the diagonal bias was explored. In each of these experiments, the subjects were explicitly instructed to adopt a diagonal-reference strategy (i.e., they were told to compare the slope of the test line in each stimulus with that of the imaginary diagonal). In Experiment 3, the stimulus displays were explicitly described as graphs, whereas in Experiment 4 they were not. If the pattern of results found for more complex graphs does reflect the use of a diagonal-reference strategy, then a bias toward the diagonal might be expected for these configurations, not only when explicitly described as graphs but also when presented simply as linear configurations.

\section{EXPERIMENT 1 \\ Perception of Slope in Simplified Graphs}

The logic of this experiment is straightforward. In our previous research, the linear configuration comprising the "structural" component of each stimulus was embedded in a display containing additional, mostly symbolic, information designed to make the stimuli compelling as graphs or maps; additional tasks were imposed to ensure appropriate interpretation. Since bias toward the diagonal was found only for configurations interpreted as graphs, the differing pattern of results for the two types of symbolic stimuli was taken to suggest that the use of the $45^{\circ}$ line as a cognitive reference frame is a strategy specific to encoding linear configurations viewed as graphs. If this is the case, then presenting the linear configurations alone, without any additional information or instructions, should also produce a different pattern of results from those found for stimuli presented as meaningful graphs. In particular, the systematic bias toward the diagonal observed for meaningful graphs either should not be found or should be considerably reduced for these stimuli. Indeed, the observation of such a difference between graph (symbolic/strategic) and "control" (structural) effects is essential to the cognitive interpretation of the previously observed graph distortions. In this experiment, as in our earlier studies, the stimulus displays consisted of fixed horizontal and vertical lines (axes) joined to make a right angle, within which the slope of a converging oblique (function) line was varied. The orientation of the oblique test line was varied from $5^{\circ}$ to $85^{\circ}$, in $5^{\circ}$ steps. The subject's task, as in our previous research, was to draw the test line on a response sheet in which the horizontal and vertical axes were given.

\section{Method}

Subjects. Thirty-five students from an introductory psychology course at Oberlin College participated as subjects in this experiment to fulfill a course requirement.

Stimuli and Design. The stimuli consisted simply of three lines: a function (test) line within a pair of orthogonal contextual axes (see Figure 1). The angle of the function line to the abscissa varied from $5^{\circ}$ to $85^{\circ}$, in $5^{\circ}$ steps, for a total of 17 stimulus angles. The length of each axis was $100 \mathrm{~mm}$, and that of the function line was $90 \mathrm{~mm}$; all lines were approximately $1 \mathrm{~mm}$ thick. All stimuli were constructed of black matte tape, centered on white paper cut square $(215 \times 215 \mathrm{~mm})$. Two copies of each stimulus were used to construct stimulus booklets. In each booklet, the stimuli were arranged in random order, alternated with colored "blank" sheets (to prevent the next stimulus from showing through).

Procedure. Each subject was given both a stimulus booklet and a response booklet. The response booklet consisted of white sheets showing bare axes (identical to those in the stimuli), alternating with blank sheets. The subjects were instructed to place the booklets on the table before them, with the stimulus booklet on their 


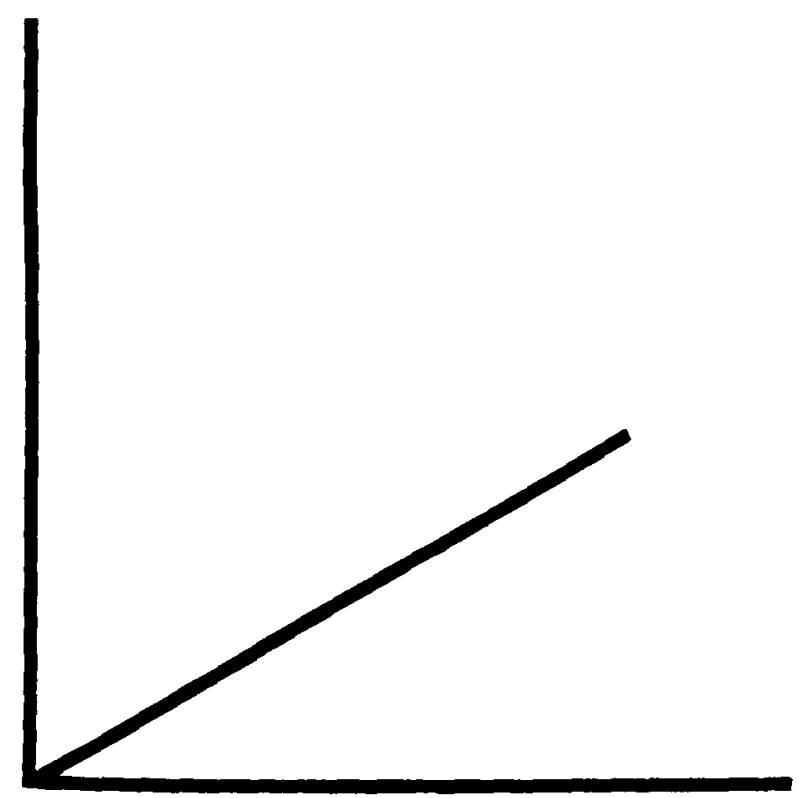

Figure 1. A sample stimulus configuration.

left and the response booklet on their right. They were asked to view each stimulus figure at their own pace and to simply draw the test line from a given stimulus on the corresponding response sheet. No erasures or corrections were permitted. In the instructions (and throughout the experimental session), stimulus and response configurations were referred to simply as "figures"; they were not described as "graphs." The subjects were run in groups of about 8-12 individuals. The entire procedure lasted about $20 \mathrm{~min}$.

\section{Results}

Using a protractor, a coder blind to the experimental conditions scored the responses by measuring the angle between the (visually estimated) best-fitting straight line through each drawn response line (beginning at the origin) and the horizontal axis. Figure 2 presents mean distortion for each stimulus angle in Experiment 1 ; the vertical lines at each point represent the standard error of the means. The function is cyclical: it begins high, then falls, rises, and falls again. Peak overestimation is found for stimulus angles of $5^{\circ}$ and $50^{\circ}$, and peak underestimation for stimuli of $30^{\circ}$ and $80^{\circ}$. The distortions at $5^{\circ}, 50^{\circ}$, and $80^{\circ}$ were highly reliable $[t(34)>2.73, p<.05$, in each case], whereas the distortion at $30^{\circ}$ was not. It should also be noted that perception of the $45^{\circ}$ line was not veridical; a significant positive distortion was found $[t(34)$ $=2.10, p<.05$ ]. An analysis of variance (ANOVA) demonstrated the reliability of the overall effect of stimulus angle $[F(16,544)=18.13, p<.05]$. Trend analyses showed significant linear, quadratic, cubic, quartic, 5 th, 7th, and 9th order effects; the largest effect was cubic $[F(1,34)=54.18, p<.05]$.

\section{Discussion}

In the perception literature, misperception of the orientation of a test line in the presence of an inducing line of a different orientation is referred to as a tilt illusion. When inducing and test lines converge and abut to form an acute angle, the lines phenomenally repulse each other in the orientation domain, an effect known as tilt contrast or acute-angle expansion. This distortion is generally reported to peak at small angles, followed by a long decline; the effect is maximized if the inducing line is either vertical or horizontal (e.g., Blakemore, Carpenter, \& Georgeson, 1970; Carpenter \& Blakemore, 1973; see also Bouma \& Andriessen, 1970; Wenderoth, Parkinson, \& White, 1979). Although the specific physiological locus of tilt-contrast effects has been the subject of some debate (see Howard, 1982), these distortions have long been most commonly attributed to the tuning characteristics of orientation-specific cortical cells in the visual system and to lateral inhibitory interactions that occur between them (Carpenter \& Blakemore, 1973; see also Blakemore, Carpenter, \& Georgeson, 1970; Wenderoth, O'Connor, \& Johnson, 1986).

The linear configurations used in Experiment 1 bear a close structural similarity to the conventional tilt-contrast illusion display. Indeed, they differ in only two respects: the graphs contain three lines rather than two, and the orientation of two of those lines is fixed at $0^{\circ}$ and $90^{\circ}$. The data on the effect of adding a third line to the standard tilt-contrast display is limited, largely confined to configurations in which the third line is placed to the far side of the induction line and is assumed to influence perception of the test line only indirectly, through "disinhibition" of the effect of the original induction line (e.g., Carpenter \& Blakemore, 1973). In general, no reliable effect has been found when the orientation of the third line differs from that of the inducing line by $45^{\circ}$ (or more) (O'Toole, 1979; see also Wenderoth \& Curthoys, 1974). However, Howard (1982) has noted the need for further research in this area, especially under conditions in which,

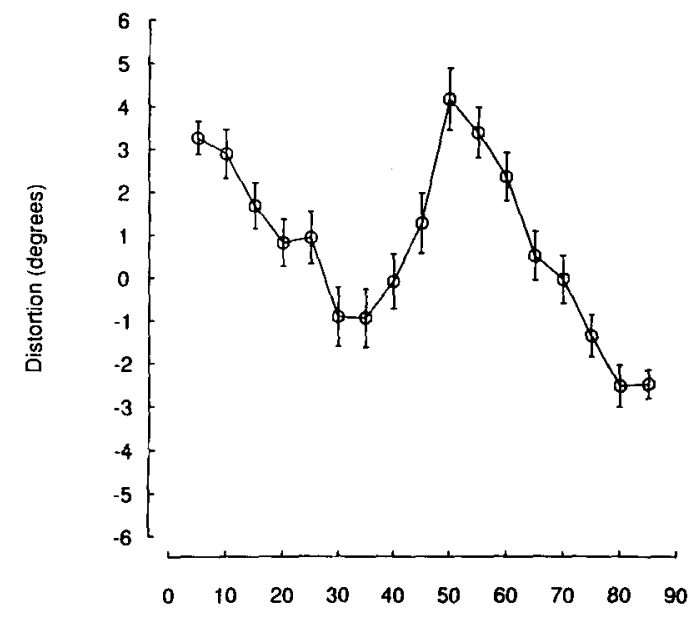

Stimulus Angle (degrees)

Figure 2. Mean distortion (in degrees) of perceived orientation as a function of stimulus angle in Experiment 1. 
as in the present experiment, the test line is placed between two inducing lines.

Recently, Wenderoth and colleagues (Wenderoth, Johnstone, \& van der Zwan, 1989; see also Wenderoth, 1977; Wenderoth \& Beh, 1977) have proposed a new way of conceptualizing tilt effects, which may prove useful in interpreting the present data. Specifically, they have argued that many tilt distortions may be profitably interpreted in terms of the major axes hypothesis, originally formulated as a general description of rod-and-frame phenomena. The major axes hypothesis (first put forward by Beh, Wenderoth, \& Purcell, 1971) attributes orientation illusions to symmetry-extraction processes assumed to take place early in perceptual organization. It predicts that a test line (or rod) within an inducing frame should appear perceptually tilted away from whichever of the frame's axes of mirror symmetry is closest to the test line. The hypothesis has been demonstrated to hold for frames of various shapes and patterns and for both physically present and "virtual" axes (e.g., Hartley, 1982; Wenderoth \& Curthoys, 1974; Wenderoth et al., 1989). The conditions of the present experiment differ substantially from those of most of that research, in which-as is characteristic of rod-and-frame studies-the test line is placed within a tilted frame and the subject's task is always to judge perceived upright of the test line (i.e., adjust or match to vertical). Yet the stimulus displays are closely related, and the predictions of the major axes hypothesis are clear.

For the conditions given in this experiment, the major axes hypothesis predicts tilt contrast against the physically present horizontal and vertical axes and also against the virtual diagonal axis of symmetry (Wenderoth, personal communication, January 1990). These predictions coincide closely with the observed results. Thus, the present findings are readily interpretable in terms of established perceptual principles. Indeed, they provide new data both on tilt effects (in which the test line is varied between two orthogonal inducing lines) and in support of the claim that the major axes hypothesis can account for both rod-andframe and tilt illusions. (For a description of the specific physiological mechanisms currently hypothesized to explain major axes effects, see Wenderoth et al., 1989). Moreover, these findings, when taken together with our previous results, demonstrate that the pattern of results for graphs differs qualitatively from that both for meaningless linear configurations (the structural component of linear graphs) and for maps (an alternative symbol system imposed upon the same structural components). Perceptual contrast was observed not only against the horizontal and vertical axes, but also against the virtual $45^{\circ}$ axis in this experiment. The results for the midrange angles (i.e., those closest to the $45^{\circ}$ line) are especially clear in demonstrating that the systematic bias toward the diagonal found for meaningful graphs is not found for meaningless linear configurations. Thus, these data provide support for the view that the use of the $45^{\circ}$ line as a cognitive reference frame is a strategy specific to viewing linear functions in graphs.

\section{EXPERIMENT 2 \\ Immediate Memory for Slope \\ in Simplified Graphs}

Conceptual biases in stimulus organization and encoding can generally be demonstrated more clearly in memorial tasks than in perceptual tasks (see, e.g., Bartlett, 1932; Bransford \& Johnson, 1972; Cermak \& Craik, 1979). Indeed, Tversky and Schiano (1989) argued that structural and strategic factors in encoding visual stimuli can often be broadly distinguished by comparing effects in perception and memory. A purely structural effect should produce similar patterns and magnitudes of distortion in perception and memory, whereas a strategic effect should show either a greater distortion in memory or a qualitatively different pattern of errors (or both). Experiment 1 demonstrated a clear pattern of perceptual distortions in viewing the structural components of linear graphs. These results differ markedly from the pattern found for immediate memory of more complex and convincing graphs, in which the direction of distortion was consistently toward the diagonal for all angles studied. This divergent pattern of findings can be explained most easily if the results of Experiment 1 are attributed to structural factors, and if the results of our earlier research are seen as reflecting the strategic use of a cognitive reference frame to anchor encoding of linear functions in graphs. Experiment 2 was designed to replicate Experiment 1 under immediatememory conditions similar to those used in our earlier research. The hypothesis described above predicts that the results of Experiment 2 should reflect only structural effects, and thus should be close in pattern and magnitude to those of Experiment 1.

\section{Method}

Subjects. Thirty-five students from an introductory psychology course at Oberlin College participated as subjects in this experiment to fulfill a course requirement.

Stimuli and Design. The stimuli and design of Experiment 2 were identical to those of Experiment 1; the response task was also the same. The procedure of Experiment 2 differed from that of Experiment 1 only in that the experimenter indicated when the subjects were to view the stimuli and make their responses. Each graph was viewed for a fixed interval of $10 \mathrm{sec}$, and then was covered with a colored blank sheet. After a 10-sec delay interval (Tversky \& Schiano, 1989, had used a 7-sec delay), the subjects were signaled to turn to the next response graph, and the drawing response was made.

\section{Results and Discussion}

Figure 3 presents mean distortion for each stimulus angle in Experiment 2. As in the results for Experiment 1, the function shows two peaks and two troughs. The stimulus angles at which peak values were obtained are highly similar to those of Experiment 1: Peak overestimation was found for stimuli of $5^{\circ}$ and $50^{\circ}$, and peak underestimation for angles of $30^{\circ}$ and $80^{\circ}$. In this experiment, the distortions at all four peaks are highly reliable $[t(34)>5.95$, $p<.05$, in each case]. Again, a significant positive distortion was found at $45^{\circ}[t(34)=2.19, p<.05]$. In- 


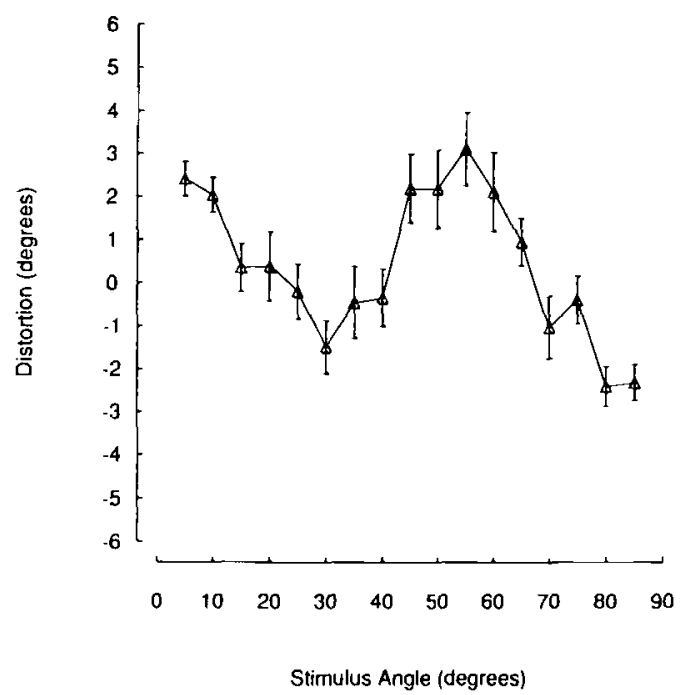

Figure 3. Mean distortion (in degrees) of remembered orientation as a function of stimulus angle in Experiment 2.

deed, the function for this experiment was remarkably close to that for Experiment 1. An ANOVA showed that the overall effect of stimulus angle was reliable in this experiment $[F(16,544)=8.72, p<.05]$. Trend analyses showed the same pattern as that found for Experiment 1: Significant linear, quadratic, cubic, quartic, 5th, 7th, and 9th order effects were found; again, the largest effect was cubic $[F(1,34)=24.47, p<.05]$. An additional ANOVA compared the overall results of Experiments 1 and 2 . As expected, there was a significant effect of stimulus angle $[F(16,1088)=23.88, p<.05]$ but no overall effect of experiment $[F(1,68)=1.49$, n.s. $]$ and no experiment $X$ stimulus angle interaction $[F(16,1088)$ $=1.14, \mathrm{n} . \mathrm{s}$.] was found. Analyses of trend components also yielded no significant differences between Experiments 1 and $2[F(1,68)<1.42$, n.s., in each case].

These results constitute a replication and extension of Experiment 1 and demonstrate the robustness of the observed distortions. The simplified graph displays used in Experiments 1 and 2 were almost identical in linear structure to the graphs used by Tversky and Schiano (those stimuli also contained axis labels and calibration "ticks"). Yet they were apparently encoded as meaningless linear configurations of the sort used in tilt illusion (and rodand-frame) research. The pattern of data again suggests perceptual contrast effects of the sort predicted by the major axes hypothesis; this "structural" interpretation is strengthened by the close similarity of the results for perception and for memory.

\section{EXPERIMENT 3 Instructional Effects}

We have argued that the systematic diagonal bias observed for remembering meaningful graphs reflects a strategy spontaneously adopted by subjects specifically to encode and remember linear functions. Support for this view was gathered in comparison conditions in our previous research, in which identical linear configurations were embedded in map displays and no evidence of a bias toward the diagonal was found. In addition, the pattern of results found for the meaningless configurations in Experiments 1 and 2 can best be explained simply in terms of perceptual contrast effects; no reference to strategic factors is required. Indeed, this is not surprising, since the stimuli in the first two experiments were simply presented as linear configurations, without any additional information or instructions to promote their being viewed as graphs. However, if the use of the diagonal as a cognitive reference frame is a strategy under cognitive control, then instructional manipulation of the subjects' viewing strategy might be expected to affect their encoding of the stimuli. In the cognitive map literature, Holyoak and Mah (1982) demonstrated that cognitive reference frames are flexible and subject to instructional manipulation: Instructing subjects to imagine themselves on the East or West coast systematically affected estimated distances between U.S. cities. In Experiment 3, the subjects were explicitly instructed to use the diagonal as a reference line in viewing the configurations. That is, while viewing each stimulus, the subjects were required to compare the slope of the test line with that of the imaginary diagonal. To further promote the use of the diagonal as a cognitive reference frame, the subjects were also explicitly told to view the stimuli as graphs. In all other ways, the stimuli and methodology of this experiment were identical to those of Experiment 2. If the results found previously for complex graphs reflect the voluntary adoption of a graphviewing strategy in which the slope of the test line is compared with that of the diagonal, then explicitly instructing subjects to perform the diagonal comparison processand to view the stimuli as graphs-should alter the pattern of results such that the diagonal bias should again be clearly observed.

\section{Method}

Subjects. Thirty-five students from an introductory psychology course at Stanford University participated as subjects in this experiment to fulfill a course requirement.

Stimuli and Design. The stimuli and design of Experiment 3 were identical to those of Experiment 2; the memory interval and response task were also the same. The procedure of this experiment differed from that of Experiment 2 only in instructions. While viewing each stimulus, the subjects were explicitly required to compare the slope of the function line with that of the implicit $45^{\circ}$ line. For each stimulus, a sheet with two questions was completed. The first question was, "Compared to the (imaginary) $45^{\circ}$ or diagonal line, the function line in this graph is: above, below, or on the diagonal." The second question was, "If the function line is not on the diagonal, it is: near to, midway to, or far from the diagonal." The subjects were familiarized with the questions prior to beginning the experiment, and they responded to each one simply by checking the appropriate answer. Thus, the comparison procedure was typically performed rapidly and did not noticeably increase stimulus viewing time. The question sheets were stapled together to form a third booklet. They, like the stimuli, were turned so as not to be visible during the memory interval. 


\section{Results and Discussion}

Figure 4 presents mean distortion for each stimulus angle in Experiment 3. The function shows that the direction of distortion was systematically driven toward the $45^{\circ}$ line. Significant overestimation was found for all angles between $5^{\circ}$ and $25^{\circ}[t(34)>4.29, p<.05$, in each case $]$, and significant underestimation was found for those between $65^{\circ}$ and $85^{\circ}[t(34)>2.40, p<.05$, in each case]. Distortion magnitude was greatest for extreme angles and fell as the $45^{\circ}$ line was approached; peak overestimation occurred for the $5^{\circ}$ stimulus, and peak underestimation for the $80^{\circ}$ stimulus (though distortion magnitude was very close for the $80^{\circ}$ and $85^{\circ}$ stimuli). No significant distortion was found at $45^{\circ}[t(34)=0.77$, n.s. $]$. An ANOVA demonstrated a significant effect of stimulus angle $[F(16,544)=$ 44.86, $p<.05$ ] in this experiment; trend analyses showed a highly reliable linear trend $[F(1,34)=98.74$, $p<.05]$. No significant higher order trend components were found.

An additional ANOVA was performed to compare the results of Experiment 3 with those of Experiment 2. Significant effects of experiment $[F(1,68)=16.56, p<.05]$ and stimulus angle $[F(1,16)=15.00, p<.05]$ were found. More interestingly, a significant experiment $x$ stimulus angle interaction $[F(16,1088)=2.43, p<.05]$ was demonstrated. Trend components showing significant differences between experiments include: quartic, 6th, 7th, 8 th, and 10th order effects $[F(1,68)>3.96, p<.05$, in each case].

In comparing the distortions for the two experiments at each stimulus angle, it is clear that there is greater distortion toward the $45^{\circ}$ line in the results of the present experiment than in those of Experiment 2. Distortion magnitude was greater in this experiment at extreme angles (between $5^{\circ}$ and $25^{\circ}$ and between $65^{\circ}$ and $85^{\circ}$ ); it was

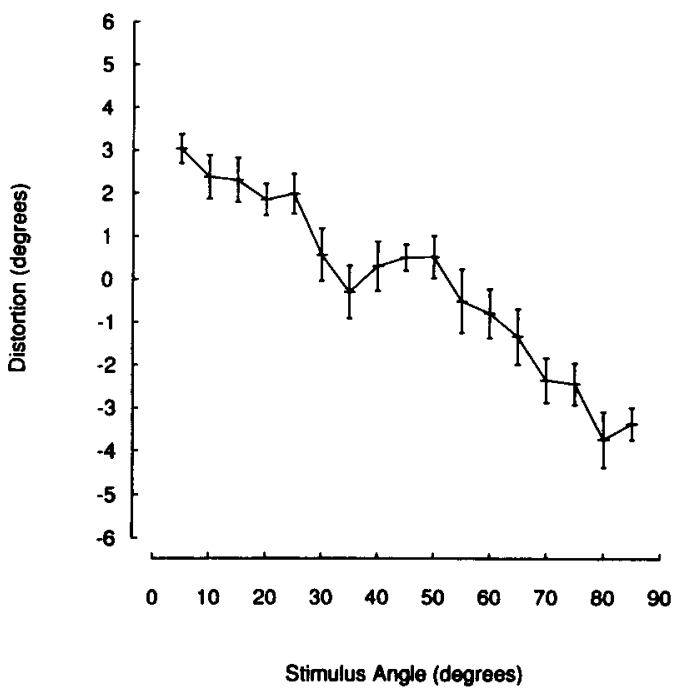

Figure 4. Mean distortion (in degrees) of remembered orientation as a function of stimulus angle in Experiment 3. greater in Experiment 2 in the midranges (between $30^{\circ}$ and $60^{\circ}$ ). An examination of the simple effects of the experiment $\times$ stimulus angle interaction shows that there was significantly greater distortion toward the $45^{\circ}$ line for 12 of the 17 stimulus angles: $15^{\circ}, 20^{\circ}, 25^{\circ}, 30^{\circ}, 45^{\circ}$, $50^{\circ}, 55^{\circ}, 60^{\circ}, 65^{\circ}, 75^{\circ}, 80^{\circ}$, and $85^{\circ}[t(34)>1.74$, $p<.05$, in each case].

The difference between the pattern of results for Experiment 3 and that for the first two experiments is striking. The function here is linear rather than cyclical, and the distortion is uniformly in the direction of the diagonal. Also, as predicted from previous work on the use of cognitive reference frames, the distortion was global and the magnitude of distortion was greatest for stimuli farthest away from the reference line (see Poulton, 1979). Trends in this direction were also found in two experiments by Tversky and Schiano (1989). Thus, the instructional manipulation used in this experiment affected the results such that they came to more closely resemble the findings observed for spontaneous encoding of more complex and convincing graphs. This provides further support for the view that the systematic bias toward the diagonal found in our previous research does indeed reflect the use of a diagonalreference strategy in encoding the slopes of linear functions in graphs. More generally, it demonstrates the power and flexibility of the use of cognitive reference frames.

\section{EXPERIMENT 4 The Diagonal-Reference Strategy}

In Experiment 3, it was shown that instructing subjects to compare the slope of the test line with that of the implied diagonal and to view the stimuli as graphs served to reinstate the pattern of bias toward the diagonal previously observed for more complex and compelling graphs. In this final experiment, subjects were again instructed to use the diagonal-reference strategy, but they were not instructed to view the stimulus configurations as graphs. If the distortion observed for meaningful graphs does reflect the use of the diagonal-reference strategy, then a similar pattern of bias toward the diagonal should be found even for these meaningless linear configurations.

\section{Method}

Subjects. Thirty-five students from an introductory psychology course at Stanford University participated as subjects in this experiment to fulfill a course requirement.

Stimuli and Design. The stimuli and design of Experiment 4 were identical to those of Experiment 3. The only difference between the two experiments is that subjects in Experiment 4 were not told to view the stimuli as graphs.

\section{Results and Discussion}

Figure 5 presents mean distortion for each stimulus angle in Experiment 4. The direction of distortion in this experiment was also systematically driven toward the $45^{\circ}$ line. Significant overestimation was found for all angles between $5^{\circ}$ and $30^{\circ}[t(34)>2.70, p<.05$, in each case], and significant underestimation was found for those between $65^{\circ}$ and $85^{\circ}[t(34)>2.62, p<.05$, in each 


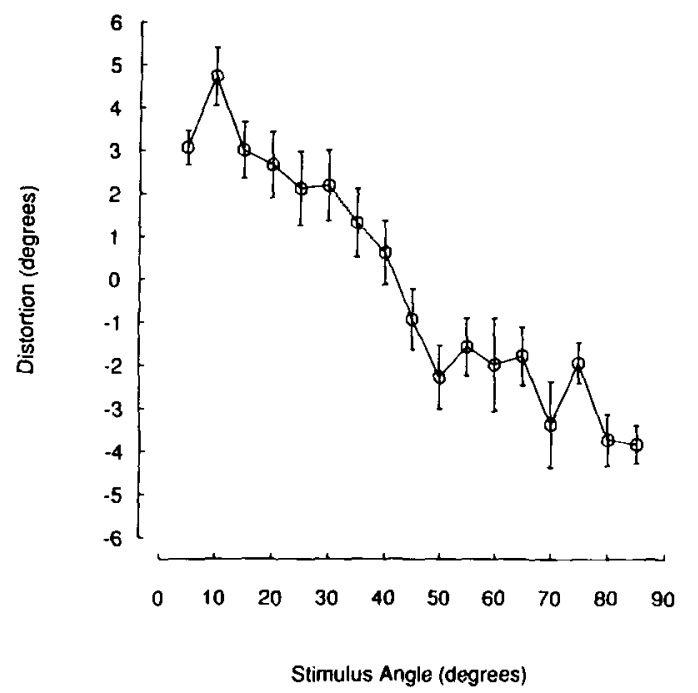

Figure 5. Mean distortion (in degrees) of remembered orientation as a function of stimulus angle in Experiment 4.

case]. No reliable distortion was found at $45^{\circ}[t(34)=$ 1.29 , n.s.]. The overall pattern found in Experiment 3, in which distortion magnitude was greatest for extreme angles and fell with proximity to the diagonal, was also observed here, although the results of Experiment 4 were clearly more variable than those of Experiment 3. Peak overestimation occurred for the $10^{\circ}$ stimulus, and peak underestimation for the $85^{\circ}$ stimulus. An ANOVA demonstrated a significant effect of stimulus angle $[F(16,544)$ $=50.08, p<.05]$ in this experiment. As in Experiment 3 , a trend analysis showed a highly reliable linear trend $[F(1,34)=109.83, p<.05]$, but no significant higher order components.

An additional ANOVA was performed to compare the results of Experiment 4 with those of Experiments 2 and 3. No overall effect of experiment was found $[F(2,102)$ $=1.34$, n.s.] for the grand means of the three experiments. More interestingly, however, a significant overall effect of stimulus angle was found $[F(16,1632)=$ $29.28, p<.05]$, as well as a significant experiment $\times$ stimulus angle interaction $[F(32,1632)=29.28, p<.05]$. This reflects significant simple interactions between Experiments 2 and $4[F(16,1088)=9.87, p<.05]$ and Experiments 3 and $4[F(16,1088)=2.37, p<.05]$, as well as the previously reported experiment $x$ stimulus angle interaction between Experiments 2 and 3. Trend analyses demonstrated significant differences between Experiments 2 and 4 in linear, quadratic, cubic, quartic, quintic, and 7 th order components $[F(1,68)>7.33, p<.05$, in each case]. Experiments 3 and 4 showed differences only in quartic and 7 th order components $[F(1,68)>3.41$, $p<.05$, in each case], which were nonsignificant in each experiment.

A comparison of Figures 2 and 4 demonstrates that the general prediction of greater distortion toward the diagonal with diagonal-strategy instructions was supported in Ex- periment 4. An analysis of the simple effects of the experiment $\times$ stimulus angle interaction indicates that significantly greater distortion toward the diagonal was found for 13 of the 17 stimulus angles: $10^{\circ}, 15^{\circ}, 20^{\circ}, 25^{\circ}, 30^{\circ}$, $45^{\circ}, 50^{\circ}, 55^{\circ}, 60^{\circ}, 65^{\circ}, 75^{\circ}, 80^{\circ}$, and $85^{\circ}[t(34)>1.80$, $p<.05$, in each case]; a marginal effect was also found at $35^{\circ}[t(34)=1.66, p=.05]$. These findings closely confirm those of Experiment 3. Moreover, they clearly indicate the effectiveness of the diagonal-reference strategy alone in producing the observed systematic bias toward the diagonal. The fact that mean distortion was higher for most stimulus angles in Experiment 4 than in Experiment 3 is difficult to explain. However, as the figures illustrate, the results of Experiment 4 were also more variable than were those of Experiment 3. Significant differences between means for these experiments were found for only two stimulus angles: $10^{\circ}$ and $50^{\circ}[t(34)>$ $2.78, p<.05$, in each case]. Thus, the diagonal-reference strategy is apparently sufficient to produce distortions similar to those observed for linear functions in graphs even for linear configurations that are not viewed as graphs.

\section{GENERAL DISCUSSION}

In four experiments, encoding of the orientation of a variable function line in orthogonal axes was examined. In the first two experiments, the stimuli were presented simply "as is" (i.e., as meaningless linear configurations), and subjects were given no instructions regarding how to view them. The drawing response was made either while still viewing the stimulus (Experiment 1 ) or after a brief delay (Experiment 2). The results of both experiments were highly similar: small angles (between about $5^{\circ}$ and $25^{\circ}$ from either axis) were generally overestimated, whereas larger angles (between about $30^{\circ}$ and $45^{\circ}$ from each axis) were generally underestimated. This pattern of results is easily interpretable in terms of Wenderoth's major axes hypothesis, which has been put forward to provide a unified explanation of such perceptual effects as the rod-and-frame and tilt illusions (Beh, Wenderoth, \& Purcell, 1971; Wenderoth, Johnstone, \& van der Zwan, 1989).

The view that judgments of size, location, and orientation can be distorted in relation to reference elements in a display has long been assumed in explanations of visual illusions (see Coren \& Girgus, 1978). While the mechanisms responsible for such distortions are not well understood, there is some evidence that factors influencing perceptual organization-such as symmetry, proximity, and so forth-may play an important role in determining whether visual distortions are found (see Coren \& Girgus, 1980; Zhang \& Schiano, 1985). The major axes hypothesis essentially assumes that axes of symmetry function as perceptual reference frames, inducing distortion of the perceived orientation of lines close to those axes. The pattern of results for the first two experiments is very similar to that of an early study by Radner and Gibson (1935), in which subjects were asked to reproduce from memory 
the tilt of a briefly flashed rotated square. Small tilts (under $30^{\circ}$ ) were overestimated, whereas larger ones (up to $45^{\circ}$ ) were underestimated.

That such perceptual factors should also influence graph-like stimuli is not surprising. Tversky and Schiano (1989) found a bias toward increased symmetry in reproductions of slightly skewed curves embedded in both graphs and maps, which (because the curves were identical in the two types of stimuli) was interpreted as a structural effect. Poulton (1985) observed a kind of acute-angle expansion when subjects read the values of points on multiple function lines in graphs; he interpreted this distortion as a variant of the classical Poggendorf illusion. The tendency to view complex displays, such as the Poggendorf and Zöllner illusions, as structural variants of the simpler tilt-contrast illusion is common (e.g., Kobayashi, 1956; Wallace, 1975; Wallace \& Crampin, 1969; see also Coren \& Girgus, 1978) but not uncontroversial (e.g., Day \& Dickinson, 1976; Hotopf, 1966, 1981; Hotopf \& Hibberd, 1989; Weintraub \& Krantz, 1971; Weintraub, Krantz, \& Olson, 1980) in the perceptual literature.

Yet a graph is more than just a linear configuration; conceptual as well as perceptual factors must be involved in graph comprehension and memory. A graph, like a map or a musical score, instantiates a specific visual symbol system with its own syntax, semantics, and pragmatics (see, e.g., Goodman, 1968). A strategy effective for encoding information presented in a graph may not be applied to a structurally similar but symbolically distinct display. Thus, in our earlier work, linear configurations presented as maps did not show the same pattern of distortion as those presented as graphs, suggesting that the diagonal-reference strategy was not employed for the map stimuli. Moreover, when, in Experiments 3 and 4, subjects were instructed to use the diagonal reference strategy, the pattern of results for the same stimuli closely resembled that found previously for meaningful graphs. The effectiveness of the diagonal-reference strategy was demonstrated by the fact that instructing subjects to use that strategy - whether the stimuli were viewed as graphs or simply as linear configurations-was sufficient for the systematic bias toward the diagonal to reappear. Viewing the stimuli as graphs did not add to the distortions produced by the diagonal-reference strategy. Thus, while the use of this strategy was inferred from the results of our previous experiments, the present findings provide more direct evidence for it.

Conceptual biases in stimulus organization and encoding have long been demonstrated in the memory literature, often as a result of instructional manipulations (see, e.g., Anderson \& Pitchert, 1978; Bartlett, 1932; Bransford \& Johnson, 1972; Brewer \& Treyens, 1981). Encoding effects are especially compelling when observed with visual stimuli, for which memory can otherwise be highly veridical (e.g., Shepard, 1967). A classic study on encoding effects in memory for visual stimuli was performed by Carmichael, Hogan, and Walter (1932), in which memory for identical forms was distorted in different ways, depending on how the form was labeled (e.g.,
O-O as "glasses" vs. "dumbbells"). Top-down or strategic effects can also result from expertise in a given domain; experts' encoding patterns have been shown to differ dramatically from that of novices in remembering visual stimuli ranging from chessboards to circuit diagrams (e.g., Chase \& Simon, 1973; Egan \& Schwartz, 1979). Similarly, applying the rules of a specific symbol system can distort memory in predictable ways, as much of the literature on cognitive maps demonstrates. Indeed, invoking distinct symbol systems can produce different patterns of errors in remembering identical stimuli: Eisenstadt and Kareev (1975) found encoding differences for the same stimuli described as representing pieces in the games of Go and Gomoku, respectively.

The present results, taken together with those of Tversky and Schiano (1989), demonstrate that conceptual biases can be introduced into the encoding of even a single line segment when it is viewed as part of a distinct symbol system such as a graph. Subjects appear to spontaneously use the (imaginary) diagonal as a cognitive reference frame for linear functions in graphs, but not for lines in maps or in meaningless linear configurations. Thus, even in encoding the orientation of a single line segment, strategic factors can produce patterns of errors in memory that are not predictable solely from the structural characteristics of the stimulus display.

\section{REFERENCES}

Anderson, R. C., \& Pitchert, J. W. (1978). Recall of previously unrecallable information following a shift in perspective. Joumal of Verbal Learning \& Verbal Behavior, 17, 1-12.

ANDrews, D. P. (1967). Perception of contour orientation in the central fovea. Vision Research, 7, 975-1013.

BARTLETT, F. C. (1932). Remembering: A study in experimental and social psychology. New York: Macmillan.

Beh, H., Wenderoth, P., Purcell, A. (1971). The angular function of a rod-and-frame illusion. Perception \& Psychophysics, 9. 353-355

Blakemore, C., Carpenter, R., Georgeson, M. (1970). Lateral inhibition between orientation detectors in the human visual system. Nature, 228, 37-39

Bouma, H., \& Andriessen, J. J. (1968). Perceived orientation of isolated line segments. Vision Research, 8, 493-507.

Bouma, H., ANDRIEsSEN, J. J. (1970). Induced changes in the perceived orientation of line segments. Vision Research, 10, 333-349.

Bransford, J. D., \& Johnson, M. K. (1972). Contextual prerequisites for understanding: Some investigations of comprehension and recall. Journal of Verbal Learning \& Verbal Behavior, 11, 717-726.

Brewer, W. F., Treyens, J. C. (1981). Role of schemata in memory for places. Cognitive Psychology, 1, 207-230.

Bryant, P. E., Somerville, S. (1986). The spatial demands of graphs. British Journal of Psychology, 77, 187-197.

BYRNE, R. W. (1979). Memory for urban geography. Quarterly Journal of Experimental Psychology, 31, 147-154.

Carmichael, L., Hogan, H. P., Walter, A. A. (1932). An experimental study of the effect of language on the reproduction of visually perceived form. Journal of Experimental Psychology, 15, 73-86.

CARPENTER, R. H. S., Blakemore, C. (1973). Interactions between orientations in human vision. Experimental Brain Research, 18, 287-303.

Cermak, L. S. , CRaIK, F. I. M. (Eds.). (1979). Levels of processing in human memory. Hillsdale, NJ: Erlbaum.

Chase, W. G., a Simon, H. (1973). The mind's eye in chess. In 
W. G. Chase (Ed.), Visual information processing. New York: Academic Press.

Cleveland, W. S. (1985). The elements of graphing data. Monterey, CA: Wadsworth.

Cleveland, W. S., McGill, R. (1985). Graphical perception and graphical methods for analyzing scientific data. Science, 229, 828-833.

Cleveland, W. S., McGill, R. (1986). An experiment in graphical perception. International Journal of Man-Machine Studies, 25. 491-500.

Coren, S., \&irgus, J. S. (1978). Seeing is deceiving: The psychology of visual illusions. Hillsdale, NJ: Erlbaum.

Coren, S., \& Girgus, J. S. (1980). Principles of perceptual organization and spatial distortion: The gestalt illusions. Journal of Experimental Psychology: Human Perception \& Peformance, 6, 404-412.

DAY, R. H., Dickinson, R. G. (1976). The components of the Poggendorf illusion. British Journal of Psychology, 4, 537-552.

EGAN, D., SCHWartz, B. (1979). Chunking in recall of symbolic drawings. Memory \& Cognition, 7, 149-158.

EISENSTADT, M., KAREeV, Y. (1975). Aspects of human problem solving: The use of internal representations. In D. A. Norman \& D. E. Rumelhart (Eds.), Explorations in cognition. San Francisco: W. H. Freeman.

GoOdMAN, N. (1968). Languages of art. New York: Bobbs-Merrill.

HARTLEY, A. A. (1982). The role of axes of symmetry in orientation illusions. Perception \& Psychophysics, 31, 367-375.

HirTle, S. C., Jonides, J. (1985). Evidence of hierarchies in cognitive maps. Memory \& Cognition, 13, 208-217.

Holyoak, K. J., MAH, W. A. (1982). Cognitive reference points in judgments of symbolic magnitude. Cognitive Psychology, 14, 328-352.

Hotopf, W. H. N. (1966). The size-constancy theory of visual illusions. British Joumal of Psychology, 57, 307-318.

Hоторғ, W. H. N. (1981). Mistracking in alignment illusions. Journal of Experimental Psychology: Human Perception \& Performance, 7, 1211-1246

Hotopf, W. H. N., \& Hibbero, M. C. (1989). The role of angles in inducing misalignment in the Poggendorf figure. Quarterly Journal of Experimental Psychology, 41A, 355-383.

HowARD, I. P. (1982). Human visual orientation. New York: Wiley.

KOBAYASHI, T. (1956). Analytical study of displacement in visual perception. Japanese Psychological Research, 3, 37-47.

KolatA, G. (1984). The proper display of data. Science, 226, 156-157.

McNamara, T. P. (1986). Mental representations of spatial relations. Cognitive Psychology, 18, 87-121.

MOAR, I., \& BowER, G. H. (1983). Inconsistency in spatial knowledge. Memory \& Cognition, 11, 107-113.

Nelson, T. O., Chaikun, S. (1980). Immediate memory for spatial location. Journal of Experimental Psychology: Human Learning \& Memory, 6, 529-545.

OTOOLE, B. I. (1979). The tilt illusion: Length and luminance changes of induction line and third (disinhibiting) line. Perception \& Psychophysics, 25, 487-496.

Poulton, E. C. (1979). Models for biases in judging sensory magnitude. Psychological Bulletin, 86, 777-803.

Poulton, E. C. (1985). Geometric illusions in reading graphs. Perception \& Psychophysics, 37, 543-548.
Radner, M., Giason, J. J. (1935). Orientation in visual perception: The perception of tip-character in forms. Psychological Monographs, 46, 48-65.

Rosch, E. (1975). Cognitive reference points. Cognitive Psychology, 7, 532-547.

SAdalla, E. K., Burroughs, W. J., a Staplin, L. J. (1980). Reference points in spatial cognition. Joumal of Experimental Psychology: Human Learning \& Memory, 6, 516-528.

SHEPARD, R. N. (1967). Recognition memory for words, sentences and pictures. Journal of Verbal Leaming \& Verbal Behavior, 6, 156-163.

STEvens, A., Coupe, P. (1978). Distortions in judged spatial relations. Cognitive Psychology, 10, 516-528.

TAYLOR, M. M. (1961). Effects of anchoring and distance perception on the reproduction of forms. Perceptual \& Motor Skills, 12, 203-230.

TUFTE, E. R. (1983). The visual display of quantitative information. Chesire, CT: Graphics Press.

Tversky, B. (1981). Distortions in memory for maps. Cognitive Psychology, 13, 407-433.

Tversky, B., \& Schinno, D. J. (1989). Perceptual and cognitive factors in distortions in memory for graphs and maps. Journal of Experimental Psychology: General, 118, 387-398.

WALLACE, G. K. (1975). The effect of contrast on the Zöllner illusion. Vision Research, 15, 963-966.

Wallace, G. K., Crampin, D. J. (1969). The effect of background density on the Zöllner illusion. Vision Research, 9, 167-177.

Weintraue, D. J., \& Krantz, D. H. (1971). The Poggendorf illusion: Amputations, rotations, and other perturbations. Perception \& Psychophysics, 10, 257-264.

Weintraud, D. J., Krantz, D. H., Olson, T. P. (1980). The Poggendorf illusion: Consider all the angles. Joumal of Experimental Psychology: Human Perception \& Performance, 6, 718-725.

WENDEROTH, P. (1977). An analysis of the rod-and-frame illusion and its variants. In R. H. Day \& G. V. Stanley (Eds.), Studies in perception (pp. 95-141). Nedlands, Australia: University of Western Australia Press.

Wenderoth, P., BeH, H. (1977). Component analysis of orientation illusions. Perception, 6, 57-75.

WENDERoTh, P., \& CuRTHOYS, I. (1974). On the non-additivity of visual tilt illusions. Quarterty Joumal of Experimental Psychology, 26, 549-555.

Wenderoth, P., Johnstone, S., van der Zwan, J. (1989). Twodimensional tilt illusions induced by orthogonal plaid patterns: Effects of plaid motion, orientation, spatial separation, and spatial frequency. Perception, 18, 25-38.

Wenderoth, P., O'Connor, T., \& Johnson, M. (1986). The tilt illusion as a function of the relative and absolute lengths of test and inducing lines. Perception \& Psychophysics, 39, 339-345.

Wenderoth, P., Parkinson, A., \& White, D. (1979). Comparison of visual tilt illusions measured by the techniques of vertical setting, parallel matching, and dot alignment. Perception, 8, 47-57.

Zhang, H. C., \& SChinNo, D. J. (1985). Experimental studies of assimilation and contrast illusions. Acta Psychologica Sinica, 16, 34-41.

(Manuscript received October 23, 1989; revision accepted for publication June 8,1991 .) 